\title{
Mass Transport and Calibration in Liquid Chromatography Particle Beam Mass Spectrometry
}

\author{
James S. Ho, Thomas D. Behymer, William L. Budde, and \\ Thomas A. Bellar \\ U.S. Environmental Protection Agency, Office of Research and Development, \\ Environmental Monitoring Systems Laboratory, Cincinnati, Ohio, USA
}

\begin{abstract}
Differences in the designs of two liquid chromatography particle beam mass spectrometry systems result in differences in the transport of ammonium acetate and differences in ion abundance-enhancing carrier effects. The effect of mobile phase composition, especially the proportion of water in the mobile phase, on transport efficiency is described. Instrument detection limits for 12 compounds with two different interface designs are presented. The calibrations are generally nonlinear explained in terms of mass transport effects and supported by experiments with isotopically labeled species that coelute with the native species. Summary results of a small multilaboratory study are presented. Calibration with isotopically labeled internal standards is recommended for real-world environmental samples. ( $)$ Am Soc Mass Spectrom 1992, 3, 662-671)
\end{abstract}

$\mathrm{T}$ The value of high performance liquid chromatography (HPLC) particle beam (PB) mass spectrometry as an analytical technique for the on-line identification of relatively low molecular weight (less than about 1000) nongas chromatographable substances is being established [1-5]. If it could be demonstrated that HPLC/PB/MS can provide simultaneous quantitative analysis with reasonable precision, accuracy, and sensitivity, this technique could find widespread application in environmental and other areas of applied analytical chemistry.

We previously conducted preliminary studies of the quantitative potential of HPLC/PB/MS $[6,7]$ and reported the discovery of a carrier process associated with the HPLC mobile phase matrix. Enhanced ion abundances were observed for a variety of polar compounds when coeluting substances or ammonium acetate were present in the mobile phase. A tendency toward nonlinear responses (positive bias) was observed for many compounds, but carriers mitigated this tendency to some extent. Subsequently other investigators both confirmed [8-12] and did not observe $[10,12]$ similar effects.

The purpose of the research reported in this paper was to study HPLC/PB/MS mass transport and calibration in detail and determine the effects of operating parameters on quantitative measurements. All results

Address reprint requests to William L. Budde, U.S. Environmental Protection Agency, Environmental Monitoring Systems Laboratory. 26 W. Martin Luther King Drive, Cincinnati, OH 45628. reported were obtained by injecting solution aliquots into a HPLC column prior to the PB/MS system. These conditions were used to simulate realistic conditions of sample analyses that require chromatographic separations.

\section{Experimental}

\section{Materials}

Compounds used in this work were the highest purity materials available from commercial sources or from the U.S. Environmental Protection Agency's (EPA) Repository for Toxic and Hazardous Materials and were used without purification. Acetonitrile was Burdick and Jackson UV grade and reagent water was obtained from a Millipore Corp. (Bedford, MA) Milli-Q water purification system. Ammonium acetate was American Chemical Society reagent grade. High-purity helium was obtained from either the U.S. Bureau of Mines or Linde, Inc. (Somerset, NJ).

\section{Instrumentation}

Two HPLC/PB/MS systems were used in this work. Most experiments were conducted with system A, which consisted of a Waters 600 MS HPLC equipped with a Waters model 712 WISP autoinjector, a Vestec (Houston, TX) model B Universal Interface, and a Vestec model 201 mass spectrometer system. Addi- 
tions to the mobile phase after the HPLC column (post-column additions) were accomplished with a LDC/Milton Roy Constametric pump and a Waters tee connection. The Vestec interface included a thermospray nebulizer, a carrier gas inlet port, a heated spray chamber, a countercurrent gas diffusion desolvation cell (CCGDDC), and a two-stage momentum separator. The thermospray vaporizer had a removable sapphire tip and the vaporizer unit was installed in the heated spray chamber with a gas-tight fitting. The desolvation chamber was constructed with a gaspermeable membrane wall, and the exterior of the membrane wall was continuously swept with a stream of dry helium in a direction countercurrent to the particle beam flow. The spectrometer control, data acquisition, and data reduction system was a Teknivent (St. Louis, MO) Vector 1. The interface design has been described in detail [13]. Experiments with post-column additions of ammonium acetate were conducted using a Vestec model A interface and a model 101 mass spectrometer, which differed slightly from the model B/201 system used for all other work with system A.

System B was a Hewlett Packard instrument consisting of a model 1090 HPLC equipped for autoinjection, a 59980 A or B particle beam interface, a model $5988 \mathrm{~A}$ (high-energy detector) or 5989A quadrupole mass spectrometer, and a RTE-A or 59940A MS Chem Station data system. Post-column additions to the mobile phase were accomplished with a Hewlett Packard model 1090 or 1050 pump and a Valco tee connection. The interface consisted of a pnenumatic nebulizer, a metal-walled desolvation chamber, and a two-stage momentum separator.

\section{Liquid Chromatography}

Most experiments were conducted using Waters Novapack $15 \mathrm{~cm} \times 2 \mathrm{~mm}$ i.d. HPLC columns packed with spherical $4-\mu \mathrm{m}$ silica particles with octadecyldimethylsilyl groups chemically bonded to the silica surface $\left(C_{18}\right.$ reverse phase column). Upchurch $C_{18}$ or Supelco guard columns or a $2-\mu \mathrm{m}$ stainless steel frit were used to protect the analytical columns. The columns were conditioned by pumping a $75 \%$ acetonitrile- $25 \%$ reagent water $(v / v)$ solution through them for several hours to remove residual impurities, column bleed, and so forth. Experiments with system $A$ in which ammonium acetate was added post-column to the mobile phase were conducted using a Supelcosil LC-18DB (5- $\mu \mathrm{m}$ particle size) $25 \mathrm{~cm} \times 4.6 \mathrm{~mm}$ i.d. reverse phase column. The mobile phase for all experiments was a mixture of acetonitrile and $0.01-\mathrm{M}$ ammonium acetate in reagent water. The solvents were degassed in an ultrasonic bath under reduced pressure and continuously purged with helium at about $30 \mathrm{~mL} / \mathrm{min}$ to maintain very low levels of dissolved air. Injection volumes were 5 to $10 \mu \mathrm{L}$ and all injections were made with autoinjectors. All through-column and post-column flow rates and gradient elutions were selected for optimum overall system performance including chromatographic performance and system sensitivity.

In experiments with system $A$ with a 2-mm-i.d. column and post-column additions of various mixtures of acetonitrile-reagent water, the flow rate was 0.3 $\mathrm{mL} / \mathrm{min}$ through the column and $0.7 \mathrm{~mL} / \mathrm{min}$ for the post-column additions. The composition of the mobile phase through the column was held at $25 \%$ acetonitrile-75\% 0.01-M ammonium acetate in reagent water $(\mathrm{v} / \mathrm{v})$ for $1 \mathrm{~min}$, then linearly programmed to $70 \%$ acetonitrile-30\% 0.01-M ammonium acetate.

In the experiments to determine instrument detection limits with system $\mathrm{A}$, the conditions described above for post-column additions of various mixtures of acetonitrile-reagent water were used with postcolumn addition of pure acetonitrile. The same conditions were used with system $B$ to determine instrument detection limits except $0.1 \mathrm{~mL} / \mathrm{min}$ of acetonitrile was added post-column.

In experiments with system A with a 4.6-mm-i.d. column and post-column additions of ammonium acetate, the flow rate of the mobile phase through the column was $0.9 \mathrm{~mL} / \mathrm{min}$ and the post-column additions of $0.1-\mathrm{M}$ ammonium acetate in reagent water were made at $0.1 \mathrm{~mL} / \mathrm{min}$. The composition of the mobile phase through the column was held at $30 \%$ acetonitrile-70\% reagent water $(v / v)$ for $1 \mathrm{~min}$, then linearly programmed to $80 \%$ acetonitrile- $20 \%$ reagent water.

All calibration experiments with systems A and B were conducted with the 2-mm-i.d. column, and the mobile phase flow rate through the column was 0.3 $\mathrm{mL} / \mathrm{min}$. With system $\mathrm{A}$ an $84 \%$ acetonitrile- $16 \%$ reagent water $(\mathrm{v} / \mathrm{v})$ mixture was added post-column to the mobile phase at $0.7 \mathrm{~mL} / \mathrm{min}$, and with system $\mathrm{B}, 0.1 \mathrm{~mL} / \mathrm{min}$ of acetonitrile was added post-column to the mobile phase. The composition of the mobile phase through the column was held constant (isocratic) at $50 \%$ acetonitrile $-50 \% \quad 0.01-\mathrm{M}$ ammonium acetate ( $v / v$ ) for all experiments except those in Figure 3 , which used the standard gradient elution.

\section{Particle Beam and Mass Spectrometry Conditions}

Three operating parameters that affected the sensitivity of system A were optimized using $200 \mathrm{ng}$ of benzidine injected without an HPLC column (flow injection) and a mobile phase of $75 \%$ acetonitrile-25\% $0.01-\mathrm{M}$ ammonium acetate in reagent water $(v / v)$. The three parameters were heat input to the vaporizer (measured at two thermocouples designated $\mathrm{T} 1$ and $\mathrm{T} 2$ ), heat input to the spray chamber, and the helium carrier gas flow rate. The three operating parameters interacted and were optimized for maximum benzidine response using a variable size simplex procedure [14]. The optimum conditions for system $A$ were $68^{\circ} \mathrm{C}$ at $\mathrm{T} 1$, responding to a controlling $\mathrm{T} 2$ at $115{ }^{\circ} \mathrm{C}$, spray chamber temperature of $65^{\circ} \mathrm{C}$, and helium carrier gas flow of 1.5 to $2.0 \mathrm{~L} / \mathrm{min}$. This simplex optimization 
procedure was repeated for each composition entering the interface during the experiments with post-column additions of various mixtures of acetonitrile-reagent water.

The helium flow in the countercurrent gas diffusion cell was $10 \mathrm{~L} / \mathrm{min}$. The two-stage momentum separator was heated to $115^{\circ} \mathrm{C}$, and reduced the pressure to about 300 torr at the first-stage rough pump and to about 0.45 torr at the second-stage rough pump. The differentially pumped spectrometer diffusion pumps maintained a pressure of $5 \times 10^{-7}$ torr in the manifold surrounding the conventional electron ionization (EI) ion source.

The desolvation chamber of system $B$ was maintained at $50{ }^{\circ} \mathrm{C}$ and the nebulizer and momentum separator somewhat warm by contact with the desolvation chamber. The He pressure to the nebulizer was set at $50 \mathrm{lb} / \mathrm{in}^{2}$, and the interface was optimized as previously described [6]. The two-stage momentum separator reduced the pressure to about 0.4 torr at the second-stage rough pump. The differentially pumped spectrometer diffusion pumps maintained a pressure of 3 to $4 \times 10^{-5}$ torr in the manifold surrounding the conventional EI ion sources.

The ion source temperatures of both systems were 255 to $290^{\circ} \mathrm{C}$, and the electron energy was $70 \mathrm{eV}$. The mass spectrometers were generally repetitively scanned from 62 to $460 \mathrm{Da}$ at $1-2 \mathrm{~s} / \mathrm{scan}$, which usually gave at least 10 scans per HPLC peak. In some work with system A the scan range was 56 to $420 \mathrm{Da}$. Some spectra were scanned below mass 62 to determine contributions from ammonium acetate. All spectra were acquired in the positive ion mode.

\section{Ion Source Tuning and Calibration}

The ion source and lens potentials were adjusted manually to optimize ion intensity from perfluorotributylamine at mass 219 while a $50 \%$ water-acetonitrile solution passed through the interface. Both spectrometers' mass scales were calibrated according to the manufacturers' procedures.

Concentration calibrations were obtained by injecting a series of concentration calibration standards into the HPLC system and collecting mass spectra as the separated components eluted from the column. The integrated (over the HPLC peak) intensities of the quantitation ions (usually the base peaks) were plotted as a function of the amount injected. Peak integrations with system A were accomplished by using data system commands to define integration limits, and automatically with system $B$ after specifying the retention time windows and a threshold value, and they were verified for reasonableness by inspection.

\section{Results and Discussion}

The two PB interfaces used in this research differed significantly in their designs and construction. System
A, which employed a thermospray nebulizer, was constructed with a CCGDDC containing a gas-permeable membrane [13]. System B, which employed a pneumatic nebulizer, was constructed with a nonpermeable metal wall desolvation chamber. The most immediate and obvious result of these differences was the appearance of the mass spectra of the analytes that were separated with 0.01-M ammonium acetate present in the mobile phase to facilitate chromatography of some analytes [7].

With system $\mathrm{A}$, the abundance of mass 60 $\left(\mathrm{CH}_{3} \mathrm{CO}_{2} \mathrm{H}^{+}\right)$from ammonium acetate was relatively low, that is, less than $10 \%$, compared to the abundances of ions from analytes that were generally present in the 100 to 500-ng range. On the other hand, with system B, which employed the nonpermeable metal wall desolvation chamber, the abundance of mass 60 was typically about a factor of 10 to 20 greater than in system $\mathrm{A}$ or 30 to $100 \%$ of the abundances of the base peaks of the analytes present in the 100 to $500 \mathrm{ng}$ range. Scanning ions below mass 62 was therefore impractical with system $B$ because of the high background at mass 60 and 61 (the ${ }^{13} \mathrm{C}$ ion of acetic acid). A mass range of 62 to $460 \mathrm{Da}$ was employed in this and all of our previous research with system B type interfaces $[6,7]$.

We attribute the differences in the abundance of mass 60 in systems $A$ and $B$ to a reduction of the ammonium acetate concentration in the CCGDDC of system A. Ammonium acetate is in equilibrium with its components in the gas and dissolved phases. As gaseous acetic acid and ammonia pass through the membrane and are removed from the system the equilibrium is reestablished by the dissociation of more ammonium acetate. Because the CCGDDC has a path length of over $3 \mathrm{~m}$, the ammonium acetate is largely depleted from the PB before it reaches the ion source:

$$
\mathrm{NH}_{4}^{+}+\mathrm{OAc}^{-} \rightleftarrows \mathrm{NH}_{3}+\mathrm{HOAC}
$$

Previously [7] we reported that ammonium acetate in the mobile phase caused significant enhancements in the ion abundances of certain analytes when observed with a system $B$ type of interface. These enhancements were by factors of 1.2 to 5.7 and were beyond those attributable to improved chromatographic peak shape and resolution. These enhancements were rationalized as facilitated transmission of the analytes through the interface by creation of more massive particles and reduced evaporation of analytes from the particles as a result of the formation of molecular aggregates by ion-dipole interactions between ammonium acetate and the analytes (carrier effect).

Because ammonium acetate is largely removed from the aerosol spray in the CCGDDC membrane interface (system $A$ ), it might be predicted that ion abundance enhancements would not be observed with system A. Figure 1 shows comparisons of the mean integrated 


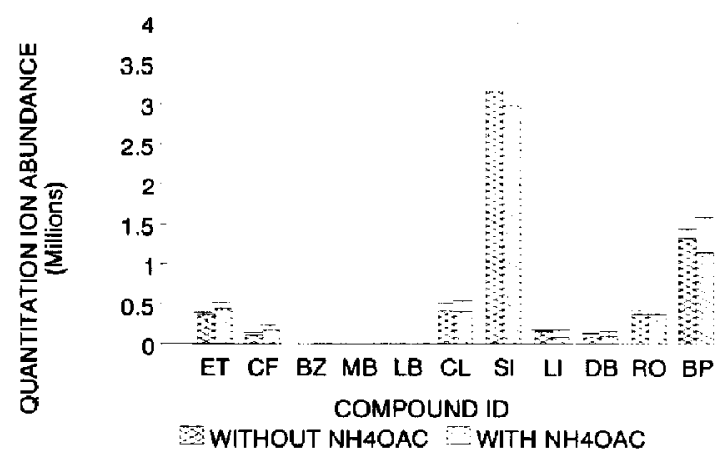

Figure 1. Comparisons of the mean integrated quantitation ion abundances of eight analytes (abbreviations in Table 1) measured with system $A$ without and with ammonium acetete added post-column to the mobile phase.

quantitation ion abundances of eight analytes (abbreviations in Table 1) measured with system A without and with post-column addition of ammonium acetate. Three analytes (three of the benzidines) could not be compared because they appear in the chromatograph as broad weak peaks without ammonium acetate in the mobile phase [7]. Ammonium acetate was not included in the mobile phase during chromatography to eliminate signal enhancements caused purely by improved chromatography. Each bar in Figure 1 represents the mean of five replicate injections of each analyte in the amounts of Table 1 (experiment 1), which also gives the names and abbreviations of the analytes and the quantitation ions. The analytes are shown in Figure 1 in elution order from left to right, which corresponds to an increasing proportion of acetonitrile to water in the mobile phase during the gradient elution. The error bars in Figure 1 are plus or minus three standard deviations around the mean.

The mean quantitation ion abundances of the applicable analytes were tested for the hypothesis of enhancement by post-column addition of ammonium acetate using the one-sided Student's t-test. Except for ethylene thiourea (ET) and caffeine (CF), which eluted during the early part of the chromatogram, there were no significant differences in the means, and therefore no evidence of enhancement at the $99 \%$ confidence level. The two early eluters showed small enhancements by factors of 1.2 (ET) and 1.5 (CF). During the early part of the gradient elution the composition of the mobile phase is more than $50 \%$ water, which tends to retain the very soluble ammonium acetate during the aerosol generation; this favors the ammonium acetate carrier effect. Ammonium acetate is only slightly soluble in acetonitrile and is more rapidly depleted in the system A interface, with the result that no carrier effect is observed for the later eluting analytes.

\section{Effect of Mobile Phase Solvent Composition}

The benefits of post-column addition of acetonitrile on the chromatographic performance and the quality of the thermospray spectra of glutathione conjugates have been reported [15]. We have observed superior PB system stability in terms of the precision of integrated ion abundance measurements when the mobile phase composition entering the PB interface does not exceed

Table 1. Analytes in elution order, quantitation ions, and amounts injected in studies of post-column additions of ammonium acetate (experiment 1) and acetonitrile-water mixtures (experiment 2) with system A

\begin{tabular}{|c|c|c|c|c|c|}
\hline \multirow[b]{2}{*}{ Analyte } & \multirow{2}{*}{$\begin{array}{l}\text { Abbre- } \\
\text { viation }\end{array}$} & \multirow[b]{2}{*}{$M W^{a}$} & \multirow{2}{*}{$\begin{array}{c}\text { Base peak and } \\
\text { quantitation } \\
\text { ion }\end{array}$} & \multicolumn{2}{|c|}{$\begin{array}{l}\text { Amount injected } \\
\text { (ng) }\end{array}$} \\
\hline & & & & Experiment $1^{b}$ & Experiment $2^{\circ}$ \\
\hline Ethylene thiourea & ET & 102 & 102 & 400 & 320 \\
\hline Caffeine & $\mathrm{CF}$ & 194 & 194 & 160 & 142 \\
\hline o-Chlorophenylthiourea & PT & 186 & 151 & $-{ }^{d}$ & 326 \\
\hline Benzidine & $B Z$ & 184 & 184 & $400^{e}$ & 229 \\
\hline 3,3'-Dimethoxybenzidine & MB & 244 & 244 & $360^{\circ}$ & 317 \\
\hline 3,3'-Dimethylbenzidine & LB & 212 & 212 & $288^{\circ}$ & 314 \\
\hline Carbaryl & $\mathrm{CL}$ & 201 & 144 & 1068 & $-\mathbf{d}$ \\
\hline Diuron & DI & 232 & 72 & $-^{d}$ & 200 \\
\hline Siduron & SI & 232 & 93 & 246 & 279 \\
\hline Linuron & L.I & 248 & 61 & 1080 & $-\mathbf{a}$ \\
\hline 3,3'-Dichlorobenzidine & $\mathrm{DB}$ & 252 & 252 & 200 & 201 \\
\hline Rotenone & RO & 394 & 192 & 200 & 500 \\
\hline Benzoylprop ethyl & $\mathrm{BP}$ & 365 & 105 & 80 & 318 \\
\hline
\end{tabular}

\footnotetext{
a Monoisotopic molecular weight calculated from the atomic masses of the isotopes with the smallest atomic masses.

Amounts injected in post-column additions of ammonium acetate.

Amounts injected in post-column additions of acetonitrile-reagent water mixtures.

Not included in this experiment.

Analytes appear in chromatography as broad weak peaks without ammonium acetate in the mobile phase.
} 
about $70 \%$ water. As with the glutathione conjugates [15], post-column addition of acetonitrile permits beginning a gradient elution with a high proportion of water (about $95 \%$ ) in the mobile phase to allow maximum chromatographic performance with very polar compounds, yet maintains good PB stability and ion abundance measurement precision.

A series of experiments was conducted with system A to determine the optimum composition of the postcolumn solvent mixture for various analytes. Using a linear gradient elution from $30 \%$ acetonitrile-70\% $0.01-\mathrm{M}$ ammonium acetate to $70 \%$ acetonitrile-30\% $0.01-\mathrm{M}$ ammonium acetate through the column at 0.3 $\mathrm{mL} / \mathrm{min}$, pure reagent water, pure acetonitrile, and two water-acetonitrile mixtures were added postcolumn at $0.7 \mathrm{~mL} / \mathrm{min}$ during the chromatography of 11 analytes. The analytes are shown in Table 1 and the amounts injected are listed under experiment 2 . Figure 2 shows comparisons of the mean integrated quantitation ion abundances of the 11 analytes with the varying compositions of the post-column addition mixture. The analytes are shown in Figure 2 in elution order from left to right, which also corresponds to increasing acetonitrile in the gradient through the column.

The precision (three replicate determinations) of these measurements varied with the composition of the mobile phase entering the interface. With addition of $100 \%$ acetonitrile post-column, the relative standard deviations (RSD) of the three measurements averaged $5.5 \%$ and only two exceeded $10 \%$ (ET and BP). With increasing water content entering the interface from either the early part of the gradient elution or as a result of post-column addition of pure reagent water or both, the precision deteriorated significantly and RSDs with $100 \%$ post-column water addition averaged 19.5\%. Nevertheless the trend in Figure 2 is toward significantly higher integrated ion abundances with increasing acetonitrile content entering the interface until about the midpoint of the gradient elution where more acetonitrile caused no additional enhancements and actually reduced the signal for several analytes at the end of the gradient elution (very high acetonitrile).

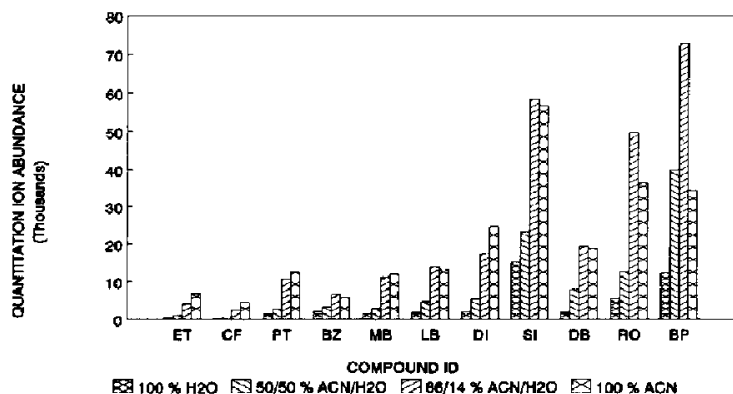

Figure 2. Effect of post-column mobile phase composition on the mean integrated quantitation ion abundances of 11 analytes measured with system $A$. The compositions in the legend (ACN is acetonitrile) are for the post-column additions, which represent $70 \%$ of the total flow to the PB interface.
Some of the enhancements shown in Figure 2 are by factors of 10 or more; these are attributed to more efficient transmission of the analytes through the interface when the composition of the mobile phase entering the interface is relatively low in water content. At relatively high water levels, condensation to the walls of the system A spray chamber is relatively high and analytes are lost [16]. As the mobile phase becomes richer in acetonitrile, particles are more abundant and smaller and condensation is reduced with resultant superior transmission of the analytes through the interface. In flow injection experiments, maximum transmission of caffeine through a system $A$ interface and minimum appearance of caffeine in the condensate was observed at about $75 \%$ acetonitrile [16].

We observed a similar but less pronounced trend with system B, but it could not be studied over the same range of solvent compositions because the amount of solvent that could be added post-column was limited to about $0.1 \mathrm{~mL} / \mathrm{min}$ and the total flow into the interface was limited to about $0.4 \mathrm{~mL} / \mathrm{min}$. We concluded that post-column addition of acetonitrile is effective and have adopted this as standard practice for the analytes in Table 1 and analogous compounds.

\section{Detection Limits}

The instrument detection limit (IDL) is defined as the amount required to give a mean integrated quantitation ion abundance three times the average of the background signal. Table 2 contains the IDLs of the analytes in Table 1 measured with systems A and B and RSDs of replicate measurements. These values were determined using standard gradient elution conditions with a 2-mm-i.d. reverse phase column in place and post-column addition of acetonitrile. The IDLs are subject to variation depending on specific conditions

Table 2. Instrument detection limits (IDL) and relative standard deviations (RSD) for replicate measurements of the analytes listed in elution order

\begin{tabular}{lrrrrr}
\hline & \multicolumn{2}{c}{ System A } & & \multicolumn{2}{c}{ System B } \\
\cline { 2 - 6 } Analyte & $\begin{array}{c}\text { IDL } \\
\text { (ng) }\end{array}$ & $\begin{array}{c}\text { RSD } \\
(\%)\end{array}$ & $\begin{array}{c}\text { IDL } \\
(\mathrm{ng})\end{array}$ & $\begin{array}{c}\text { RSD } \\
(\%)\end{array}$ \\
\hline \hline ET & 5 & 3.6 & 6 & 2.2 \\
CF & 5 & 4.8 & 1 & 3.2 \\
PT & 60 & 12.2 & 7 & 4.0 \\
BZ & 20 & 7.0 & 4 & 6.2 \\
MB & 10 & 4.9 & 6 & 6.6 \\
LB & 10 & 5.6 & 2 & 5.9 \\
CL & 100 & 5.0 & 30 & 4.9 \\
SI & 1 & 3.1 & 2 & 2.7 \\
LI & 100 & 4.4 & 130 & 7.6 \\
DB & 5 & 7.7 & 3 & 3.1 \\
RO & 5 & 5.3 & 70 & 10.0 \\
BP & 5 & 8.7 & 4 & 2.4 \\
\hline
\end{tabular}


including the condition of the column, interface, and ion source.

One factor affecting the IDLs is the vapor pressure of the analyte in the interface. Carbaryl (CL) is somewhat more volatile than most of the other analytes and evaporates from the aerosol particles in the desolvation chamber; it is pumped out of the system in the momentum separator. It has relatively high IDLs on both systems, but higher on system $A$, which provides more heat input from the thermospray nebulizer. But the extra heat input in system $A$ is beneficial in giving a lower IDL for the relatively nonvolatile rotenone, which has a tendency to condense in the system $B$ interface. In a specific application, the system should be optimized for the specific compound by adjusting interface conditions. In these experiments the systems were optimized for best overall performance with all the analytes.

Another factor affecting the IDL is the availability of an abundant ion in the scan range. The base peak of linuron is at mass 61, which was not in the scan range for both systems for these particular experiments. The IDL for linuron was measured at the $5 \%$ relative abundance mass 161 ion, which is the most abundant alternative ion. Therefore the IDL for linuron is artificially high by about a factor of 20 with system A, which can normally scan mass 61 in the presence of ammonium acetate.

\section{Calibration}

Previously we reported results that suggested linear behavior for at least some analytes in the presence of ammonium acetate over certain concentration ranges [6]. Subsequent, more precise measurements indicated that nonlinear behavior was far more prevalent. In the current study calibrations for quantitative analyses were evaluated precisely with systems $A$ and $B$ by making 6 to 8 injections of various amounts of the test analytes and plotting the integrated abundances of the quantitation ions as a function of the amounts injected. Figure 3a shows calibration curves for the four benzidines (Table 1) measured with system $B$ over the 5 to $460 \mathrm{ng}$ range of injected amounts. These plots were judged nonlinear. With system $\mathrm{A}$, benzidine gave a nonlinear calibration (Figure $3 b$ ) over the range of 18 to $180 \mathrm{ng}$.

Some of the other analytes in Table 1 displayed similar behavior, some more pronounced nonlinear behavior, and a few near-linear behavior depending on the range of amounts injected. Nonlinear calibrations have also been observed for a number of carboxylic acids and several other analytes $[8,9,11,12]$. This behavior suggested that the amount of an analyte in a particle was a significant factor affecting the transmission of analytes through the interface or the detection of the analytes in the ion source. The strong tendency toward nonlinear behavior (Figure $3 a$ and $b$ ) could be

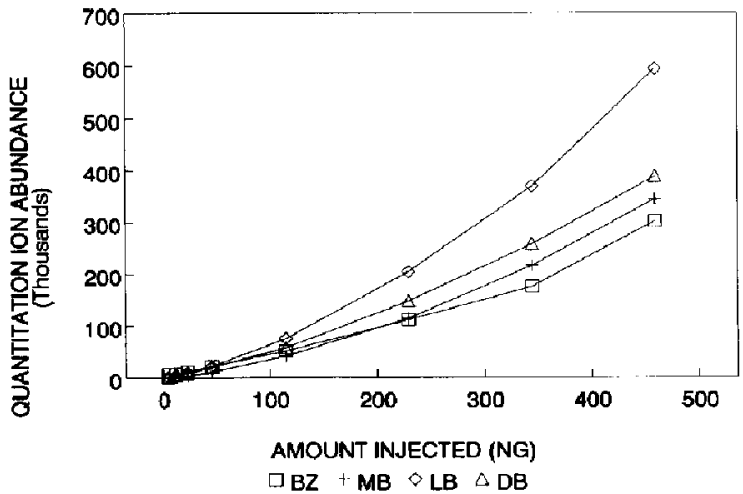

(a)

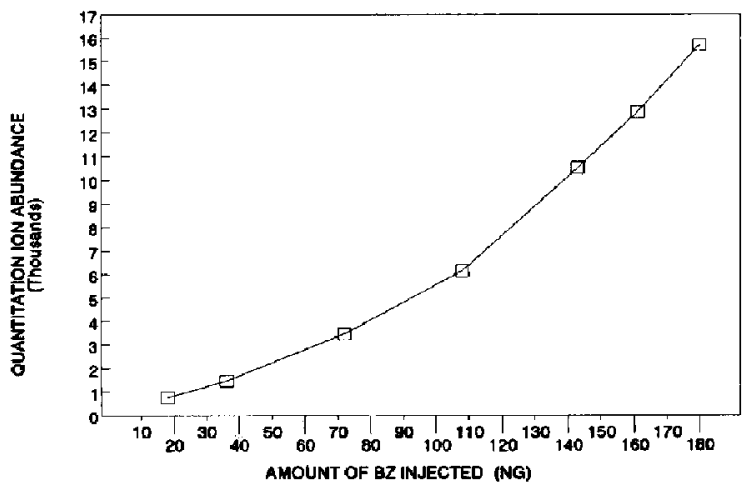

(b)

Figure 3. (a) Calibration curves for the four benzidines with system B. (b) Calibration for benzidine with system A.

rationalized as more efficient mass transport at higher analyte concentrations. This is certainly consistent with the concept [2] that particle size is a function of concentration and larger particles are transported more efficiently. To our knowledge particle size distributions have not been determined for the thermospray nebulizer used in system $A$ or the commercial nebulizer used in system $B$.

From our experience with nonlinear calibrations we surmise that at less than roughly $100 \mathrm{ng}$ injected more severe analyte losses occur through turbulence [2] or beam spreading before the skimmers in the momentum separator. Evaporation of analytes from the surface of smaller particles may also be more prevalent. But as the particle mass is increased up to and beyond some critical level, transmission of the more massive particles is more efficient and this efficiency is reasonably consistent until the interface becomes saturated with analyte. This mass transport hypothesis is also consistent with the carrier effect of ammonium acetate and coeluting substances including column bleed. Several experiments were conducted with both systems to test this hypothesis.

Figure 4 shows the calibration for deuterium-labeled benzidine $\left(D_{8} B Z\right)$ in the presence of an initially large 


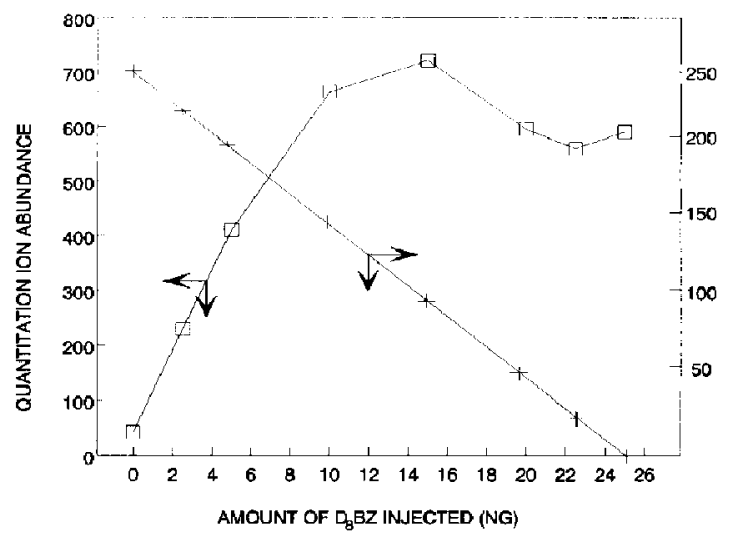

Figure 4. Calibration for deuterium-labeled benzidine with system $\mathrm{A}$ in the presence of an initially large but steadily declining excess of coeluting native benzidine.

but a steadily declining excess of coeluting native benzidine. As expected, the integrated ion abundance of $\mathrm{D}_{8} \mathrm{BZ}$ increases with the amount injected up to about $15 \mathrm{ng}$, then actually declines although the amount injected continues to increase. The point of descent of the signal corresponds to where the sum of the amounts of labeled and native benzidine injected is $70 \mathrm{ng}$. The amounts of each substance injected at each point in Figure 4 are shown under the variable sum experiment in Table 3 . Figure 4 suggests that as the masses of the particles are reduced by reducing the amount of a coeluting native benzidine, the transmission of $D_{8} B Z$ is reduced significantly. Measurements of $\mathrm{D}_{8} \mathrm{BZ}$ below the instrument detection limit of benzidine (Table 2) in this experiment were easily possible because of the enhanced transmission in the presence of excess native benzidine. Essentially identical results were obtained with system $B$.

If the mass transport hypothesis is correct, calibrations of both native and labeled analytes should be independently linear, or nearly linear, as long as the sum of the amounts injected is constant - that is, there are no changes in the masses of the particles through-

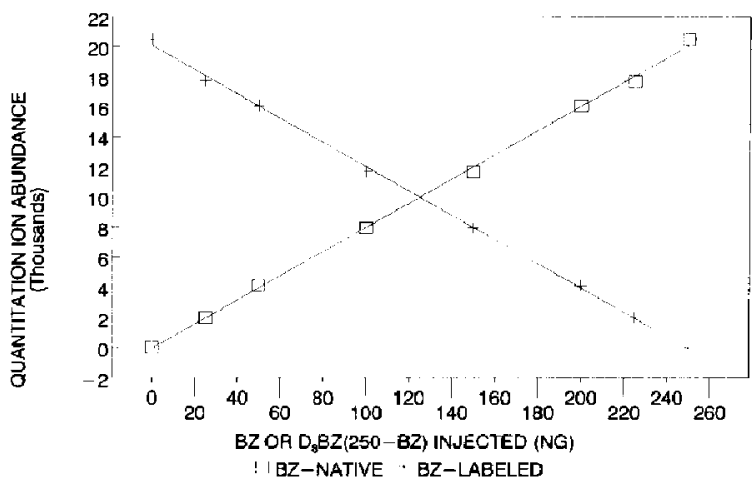

(a)

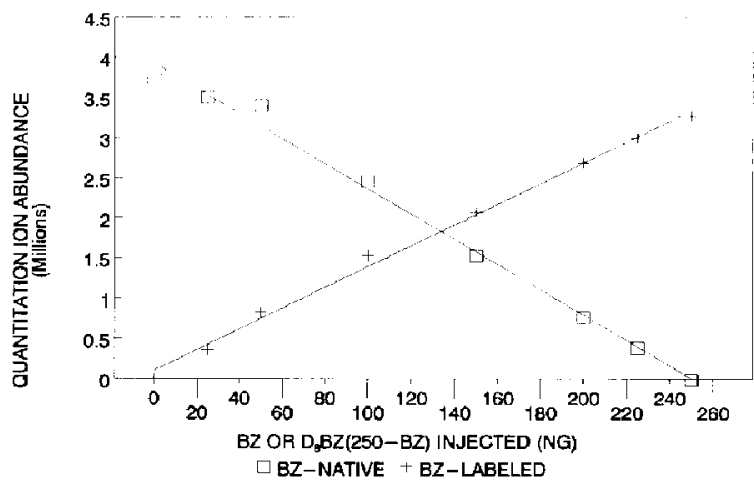

(b)

Figure 5. Calibrations for native and deuterium-labeled benzidine with (a) system $A$ and (b) system $B$, with the sum of the two analytes constant at $250 \mathrm{ng}$.

out the calibration range, and the total mass is above the critical amount. Figure 5 a shows the result of an experiment with system $A$ where the sum of $B Z$ and $\mathrm{D}_{8} \mathrm{BZ}$ injected was constant at $250 \mathrm{ng}$, but the individual amounts were varied as shown in Table 3 under the constant sum experiment. Figure $5 b$ shows the same experiment with system B. All calibrations are linear, which shows that amounts of analytes injected in $\mathrm{PB} / \mathrm{LC} / \mathrm{MS}$ significantly affects the transmission of

Table 3. Quantities in nanograms of native and deuterium-labeled benzidine injected in mass transport experiments.

\begin{tabular}{cclccrc}
\hline \multicolumn{2}{c}{ Variable sum experiment } & & \multicolumn{2}{c}{ Constant sum experiment } \\
\cline { 6 - 7 } BZ (native) & $\mathrm{BZ}\left(\mathrm{D}_{\mathrm{B}}\right)$ & Total & & $\mathrm{BZ}$ (native) & $\mathrm{BZ}\left(\mathrm{O}_{\mathrm{B}}\right)$ & Total \\
\hline 250 & 0 & 250 & 250 & 0 & 250 \\
225 & 2.5 & 227.5 & & 225 & 25 & 250 \\
200 & 5 & 205 & 200 & 50 & 250 \\
150 & 10 & 160 & 150 & 100 & 250 \\
100 & 15 & 115 & 100 & 150 & 250 \\
50 & 20 & 70 & 50 & 200 & 250 \\
25 & 22.5 & 47.5 & 25 & 225 & 250 \\
0 & 25 & 25 & 0 & 250 & 250 \\
\hline
\end{tabular}


analytes through the system. It should be noted that Figure $5 a$ and $b$ are not isotope dilution calibrations.

Similar results were obtained with 3,3'-dichlorobenzidine and caffeine, which were the only other compounds available as labeled and native species. Mass transport effects would also explain the signal enhanc. ing and linearizing effects of coeluting malic acid on daminozide [8] and coeluting phenoxyacetic on several chlorinated phenoxyacetic acids [9]. These coeluting substances added sufficient mass to the particles to exceed the critical level, and the transport efficiency remained more or less constant over the calibration range.

These experiments also suggest that coelution of small amounts of unrecognized substances in realworld environmental samples could have significant impacts on calibrations and quantitative analyses. A standard approach to calibration to compensate for variations in instrument response is the use of internal standards whose signals vary in proportion to the variations in the analyte signals. But noncoeluting internal standards did not improve linearity and will suffer from the same mass transport effects from unrecognized coeluting substances in real-world environmental samples. Unrecognized substances could cause selective signal enhancements and result in serious measurement errors. Noncoeluting internal standards offer little advantage over external standards, which are measured in a separate experiment.

On the other hand, coeluting (usually isotopically labeled) internal standards, if present in high enough quantities, could boost the total amount of analyte above the critical level. Also the ratio of the signals from the native and labeled species should be less sensitive to unrecognized coeluting substances and slight change in particle mass and/or transmission efficiency. Figure 6 shows a plot of the same data used for Figure 4 (variable sum experiment-Table 3) except that the ratio of the integrated ion abundances of native to labeled benzidine is plotted against the ratio

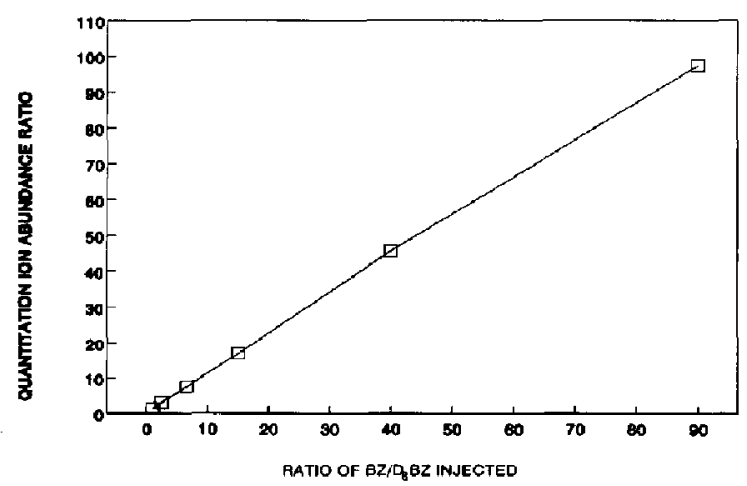

Figure 6. Isotope dilution calibration for deuterium-labeled benzidine with system $A$ in the presence of an initially large but steadily declining excess of coeluting native benzidine. of the amounts injected. The slope of this line is the usual internal standard response factor, which is nearly constant over the range of amounts injected.

A constant response factor would be expected if both species (labeled and native) were equally affected by changes in mass transport caused by changes in the sum of the amounts of coeluting species injected. Similar linear calibrations were observed with system $B$ and for $3,3^{\prime}$-dichlorobenzidine and caffeine, which were the only other labeled analytes available. Although we have not tested this approach with real-world environmental samples, this may be the only really reliable approach to quantitative analyses of real-world samples in PB/LC/MS. Recently, linear calibrations with $\mathrm{PB} / \mathrm{LC} / \mathrm{MS}$ were indicated [12] for several carboxylic acids when the ratios of responses of native and labeled species were plotted against the concentration of the native species.

\section{Multilaboratory Comparison Study}

To further test the calibration requirements and mass transport effects of $\mathrm{PB} / \mathrm{LC} / \mathrm{MS}$, a small multilaboratory study was organized. Twelve laboratories volunteered to analyze acetonitrile solutions containing each of the four benzidines in the 5 to $100-\mu \mathrm{g} / \mathrm{mL}$ concentration range. The exact concentrations were unknown to the laboratories, but each was provided with calibration solutions, a quality control check solution of known concentrations, and a draft analytical method containing step-by-step procedures (EPA Method 553). Unknown to the laboratories was the presence of equivalent concentrations of $\mathrm{D}_{8} \mathrm{BZ}$ and $\mathrm{D}_{6} \mathrm{DB}$ in some of the solutions. The purpose of the latter was to determine whether signal enhancements from coeluting substances would be observed in a multilaboratory study. The instrumentation used by the laboratories included pneumatic, heated pneumatic, thermospray, and combined pneumatic-ultrasonic [17] nebulizers. Also both impermeable and semipermeable membrane desolvation chambers were used as components of five different commercial PB/LC/MS systems. External standardization and second-order regression was the recommended calibration procedure. The unknowns were in the form of five Youden pairs [18].

Table 4 shows the mean recoveries of the four analytes at 10 and $100 \mu \mathrm{g} / \mathrm{mL}$, the multilaboratory precision (RSD) for each analyte, and an estimate of the single analyst RSD obtained from the differences in the measurements of the Youden pairs [18], which did not contain deuterated compounds. These data were obtained from the regression equations of the statistical analysis of the results of the multilaboratory study. A more complete description of the study design and a statistical analysis of the study may be published elsewhere [19].

Recoveries were excellent for all four analytes, demonstrating that very good quantitative accuracy is 
Table 4. Mean recoveries, multilaboratory precision, and estimates of single analyst precision (relative standard deviation, RSD) for the measurements of four benzidines by particle beam liquid chromatography mass spectrometry

\begin{tabular}{|c|c|c|c|c|c|c|}
\hline \multirow[t]{3}{*}{ Compound } & \multicolumn{3}{|c|}{$10 \mu \mathrm{g} / \mathrm{mL}$} & \multicolumn{3}{|c|}{$100 \mu \mathrm{g} / \mathrm{mL}$} \\
\hline & \multirow[b]{2}{*}{$\begin{array}{c}\text { Recovery } \\
(\%)\end{array}$} & \multicolumn{2}{|c|}{ RSD } & \multirow[b]{2}{*}{$\begin{array}{c}\text { Recovery } \\
(\%)\end{array}$} & \multicolumn{2}{|c|}{ RSD } \\
\hline & & $\begin{array}{c}\text { Multi } \\
\text { laboratory }\end{array}$ & $\begin{array}{c}\text { Single } \\
\text { analyst }\end{array}$ & & $\begin{array}{c}\text { Multi } \\
\text { laboratory }\end{array}$ & $\begin{array}{c}\text { Single } \\
\text { analyst }\end{array}$ \\
\hline$\overline{B Z}$ & 96 & 10 & 5.6 & 97 & 10 & 9.1 \\
\hline MB & 104 & 20 & 18 & 95 & 10 & 7.0 \\
\hline LB & 98 & 14 & 10 & 97 & 8.6 & 4.9 \\
\hline DB & 96 & 18 & 9.4 & 97 & 9.1 & 4.6 \\
\hline
\end{tabular}

possible with PB/LC/MS when the samples do not contain unexpected coeluting substances. Single analyst precision was estimated at 10\% RSD or less for all analytes except the low-concentration $\mathrm{MB}$, and overall precision was $20 \%$ RSD or less for all analytes. These data are very encouraging and of the same order of magnitude as might be expected in a similar study design using capillary column gas chromatography/ mass spectrometry.

Two solutions analyzed by the laboratories contained approximately 30 and $40 \mu \mathrm{g} / \mathrm{mL}$ of the analytes. Two other solutions contained the same analytes at the same concentrations and equivalent concentrations of $D_{8} B Z$ and $D_{6} D B$. The differences in the reported concentrations of native $\mathrm{BZ}$ and native $\mathrm{DB}$ in the solutions with and without the labeled compounds were tested for significant enhancement using Student's $t$-test. For $B Z$ there was a significant enhancement in the multilaboratory data at the $95 \%$ confidence level but not at the $99 \%$ level. For DB there was a significant enhancement at both the 95 and $99 \%$ confidence levels. Therefore the effect of enhancement from coeluting substances was observed in the multilaboratory study even though the coeluting substance was at about the same concentration as the analyte.

\section{Conclusion}

The efficiency with which analytes are transported through a PB interface depends at least on the design of the interface, the nature of the mobile phase, the vapor pressure of the analyte, the concentration of the analyte, and the presence of coeluting carrier substances. Single and multiple laboratory studies have shown that calibration with external standards and a second-order regression curve can give very good recoveries and reasonable precision when the samples are free from unexpected coeluting substances. But the presence of unexpected coeluting substances in realworld samples will cause calibration errors and analytical bias. This approach may be used with real-world samples, but analytes must ascertain that signal enhancements from coeluting substances are compensated in the external calibration standards and that no unexpected coeluting substances are in the samples.
The most reliable calibration method for real-world environmental samples is the use of coeluting isotopically labeled internal standards. These calibrations are linear because increases in the amount of native analyte injected increase the transport efficiency of both the coeluting labeled and native species to about the same extent and compensate for the mass transport effect. Unexpected coeluting substances in real-world samples should also affect the transport of the native and labeled species to about the same extent. Isotope dilution calibration is limited by the availability and cost of the labeled species and the requirement of coelution. We have observed that some labeled compounds, for example $D_{10}$-pyrene, are resolved from the native species under the LC conditions used in this work.

\section{Disclaimer}

Mention of trade names or commercial products does not constitute endorsement or recommendation for use.

\section{Acknowledgment}

The authors thank John Wilkes of the Vestec Corporation for an advanced copy of his Ph.D. thesis, University of Houston [16].

\section{References}

1. Willoughby, R. C.; Browner, R. F. Anal. Chem. 1984, 56, 2626-2631.

2. Winkler, P. C.; Perkins, D. D.; Williams, W. K.; Browner, R. F. Anal. Chem. 1988, 60, 489-493.

3. Budde, W. L.; Behymer, T. D.; Bellar, T. A.; Ho, J. S. I. Am. Water Works Assoc. 1990, 82, 60-65.

4. Voyksner, R. D.; Smith, C. S.; Knox, P. C. Biomed. Environ. Mass Spectrom. 1990, 19, 523-534.

5. Kim, I. S.; Sasinos, F. I.; Stephens, R. D.; Brown, M. A Environ. Sci. Technol. 1990, 24, 1832-1836.

6. Behymer, T. D.; Bellar, T. A.; Budde, W. L. Anal. Chem. 1990 , $62,1686-1690$.

7. Bellar, T. A.; Behymer, T. D.; Budde, W. L. J. Am. Soc. Mass Spectrom. 1990, 1, 92-98.

8. Kim, I. S.; Sasinos, F. I.; Stephens, R. D.; Brown, M. A. I. Agric, Food Chem. 1990, 38, 1223-1226

9. Mattina, M. J. I. J. Chromatogr. 1991, 554, 385-395. 
10. Doerge, D. R.; Miles, C. J. Anal. Chem. 1991, 63, 1999-2001.

11. Apffel, A.; Perry, M. L. J. Chromatogr. 1991, 554, 103-118.

12. Brown, F. R.; Draper, W. M. Bio. Mass Spectrom. 1991, 20, 515-521.

13. Vestal, M. L.; Winn, D. H.; Vestal, C. H. In LC-MS: New Developments and Applications to Pesticide, Pharmaceutical and Environmental Analysis; Brown, M. A., Ed.; ACS Symposium Series, American Chemical Society, Washington, DC, 1990; p. 215-231.

14. Morgan, S. L.; Deming, N. Anal. Chem. 1974, 46, 1170-1181.
15. Bean, M. F.; Pallante-Morell, S. L.; Dulik, D. M.; Fenselau, C. Anal. Chem. 1990, 62, 121-124.

16. Wilkes, J. G.; Ph.D. Thesis, University of Houston, Houston, TX; November 1991.

17. Ligon, W. V.; Dorn, S. B. Anal. Chem. 1990, 62, 2573-2580.

18. Youden, W. J.; Steiner, E. H. Statistical Manual of the Association of Official Analytical Chemists; Association of Official Analytical Chemists: Washington, DC; 1975.

19. Longbottom, J.; Edgell, K., private communication, August 1991. 